\title{
HUGO 20th anniversary lecture
}

\section{Genomics: 20 years ago and 20 years from now}

\section{Charles R. Cantor}

SEQUENOM, Inc., San Diego, CA, United States of America

In 1988 the vision of mapping and sequencing entire genomes was well established but the techniques that would actually be employed to achieve this were still under development. It was realistic to expect that the goals of the human genome project, the "complete" sequence of the human genome and several model organism genomes would be accomplished in less than two decades. However, no one could predict the speed at which technology would advance and lead to the continual exponential growth of genomic sequence data. The genomes of higher organisms have produced at least two surprises: the high degree of copy number variation, and the incredible diversity and importance of DNA methylation. Thus much remains to be done to convert the abundant reference genome sequences into templates that allow the reliable prediction of genome function.

In 2028 I am quite confident that DNA sequence information will play an important role in the management of individual healthcare, but I am less certain about how this will evolve from knowledge of loci that play an important role for populations to methods for using these loci to make effective predictions for individuals. While it will be technically feasible by then to sequence every individual in a cost effective way, how to use this information to guide individual healthcare and life style decisions seems to be a much more difficult problem than obtaining the information. My own personal hunch is that epigenetic data, gene expression patterns, copy number variations, metagenomic data on human symbionts and parasites, protein and small molecule profiling will play a larger role than basic variations in DNA sequence. This is unfortunate because such data is more challenging to obtain than basic genomic DNA sequence. 